\title{
Uso de Hipotermia en el Paro Cardiorrespiratorio Extrahospitalario: Reporte de un Caso
}

\author{
RAÚL BUSTOS B. ${ }^{1}$, GONZALO SOTO G. ${ }^{1}$, AMPARO SÁNCHEZ Z. ${ }^{2}$, MARIANA MONTOYA S. ${ }^{2}$ \\ 1. Unidad de Cuidados Intensivos Pediátricos, Hospital Guillermo Grant Benavente, Concepción-Chile. \\ 2. Becadas de Pediatría, Universidad de Concepción.
}

\begin{abstract}
Use of Hypothermia in Out of Hospital Pediatric Cardiac Arrest: A Case Report

Moderate hypothermia decreases mortality and improves neurological outcome in adults as well as newborns affected with Neonatal Asphyxia. The effect of hypothermia among children with cardiac arrest (CRA) is not known. Objective: Case description of the use of therapeutic hypothermia in a pediatric patient with cardiac arrest. Clinical Case: Four-year-old girl who presented extra-hospitalary CRA, where moderate hypothermia (34 degrees Celsius) was used for neurological protection in a PICU. The patient was discharged in satisfactory neurological condition, as rated through a Pediatric Cerebral Performance Category (PCPC) Test assessment, with a score of 2, similar to her baseline evaluation. Use of Hypothermia in PICU is discussed, as well as methods to induce this state and potential side effects of the treatment. (Key words: Hypothermia, pediatrics, cardiac arrest, neurological injury).

Rev Chil Pediatr 2009; 80 (6): 545-550
\end{abstract}

\section{RESUMEN}

La hipotermia moderada mejora la mortalidad y el pronóstico neurológico en el paro cardíaco en adultos y en recién nacidos con asfixia neonatal. El efecto de la hipotermia en niños con paro cardiorrespiratorio (PCR) no se conoce. Objetivo: Describir el uso de la Hipotermia terapéutica en el PCR en una paciente pediátrica, y actualizar el conocimiento en dicha materia. Caso clínico: Preescolar sexo femenino, 4 años de edad que presentó un PCR extra hospitalario en la que se uso como neuroprotección hipotermia moderada $\left(34{ }^{\circ} \mathrm{C}\right)$ durante 48 horas en la unidad de cuidados intensivos pediátricos, lo que permitió su alta en una condición neurológica satisfactoria, estimada por la escala Pediatric Cerebral Performance Category (PCPC) de 2, similar a su condición basal. También se revisa el uso de hipotermia en cuidado intensivo pediátrico, los métodos para inducir hipotermia y potenciales efectos secundarios de esta terapia.

(Palabras clave: Hipotermia, pediatría, paro cardiorrespiratorio, daño neurológico).

Rev Chil Pediatr 2009; 80 (6): 545-550

Trabajo recibido el 20 de julio de 2009, devuelto para corregir el 05 de agosto de 2009, segunda versión el 21 de agosto de 2009, aceptado para publicación el 17 de septiembre de 2009.

Correspondencia a:

Dr. Raúl Bustos B.

E-mail: robustos64@yahoo.com 


\section{Introducción}

El paro cardiorrespiratorio (PCR) en niños constituye un evento catastrófico que es asociado con una alta mortalidad y secuelas neurológicas ${ }^{1-5}$.

Las intervenciones destinadas a mejorar la encefalopatía hipóxico isquémica (EHI) que se produce posterior a un PCR han sido investigadas por décadas. En dos ensayos clínicos randomizados en adultos con fibrilación ventricular, la aplicación de hipotermia terapéutica (HT) demostró mejorar la sobrevida y el pronóstico neurológico ${ }^{6-7}$. Por otro lado, tres ensayos de HT realizados en recién nacidos con EHI también demostraron mejoría en la sobrevida y secuelas neurológicas ${ }^{8-10}$. Sin embargo, hasta la fecha no se han realizado ensayos clínicos de HT en el PCR en niños.

El objetivo de la presente comunicación es reportar el caso de una paciente de cuatro años ingresada a nuestra Unidad de Cuidado Intensivo Pediátrico (UCIP) en coma después de un PCR donde la reanimación precoz y agresiva y el uso de hipotermia moderada a $34^{\circ} \mathrm{C}$ por $48 \mathrm{~h}$ se asoció a una evolución neurológica favorable. Además se presenta una revisión del uso de hipotermia en pacientes pediátricos críticos, los métodos disponibles para inducir hipotermia y los potenciales efectos adversos de esta terapia.

\section{Caso Clínico}

Paciente de 4 años, sexo femenino, con antecedentes de hipotonía de origen no establecido por abandono de controles, que evolucionó con un cuadro de fiebre tos productiva y disnea de 24 horas de duración. De camino a nuestro hospital presentó compromiso de conciencia agudo por lo que es trasladada por personal policial al servicio de urgencia en paro cardiorrespiratorio. Al ingreso en la sala de emergencia, se constató como ritmo cardíaco asistolia, realizándose intubación endotraqueal, masaje cardíaco externo y se administró 3 dosis de adrenalina por vía periférica. Se observó retorno de la circulación espontánea a los 12 minutos de iniciado el soporte vital avanzado. Poste- riormente, en la UCIP el examen de ingreso reveló: escala neurológica de Glasgow 6, FC $127 \mathrm{pm}$ en ritmo sinusal, presión arterial 57/31/ $36 \mathrm{mmHg}, \mathrm{SaO}_{2} 85$, temperatura $\mathrm{T}^{\mathrm{o}} 36,5^{\circ} \mathrm{C}$, extremidades frías y llene capilar de 5 segundos. Disminución de murmullo vesicular en hemitórax derecho. La paciente se conectó a ventilación mecánica y se administró volumen, se inició infusión de dobutamina a través de un catéter venoso central, sedo-analgesia con midazolam-fentanilo y antibióticos biasociados. Entre los exámenes al ingreso a UCIP destacaban: glicemia $416 \mathrm{mg} / \mathrm{dL}$, creatinina $0,8 \mathrm{mg} / \mathrm{dL}$, lactato $19,4 \mathrm{mmol} / \mathrm{L}, \mathrm{pH} 6,81 \mathrm{~EB}-22, \mathrm{ScvO}_{2}$ $62 \%$. Recuento de leucocitos $1600 / \mathrm{mm}^{3}$ y plaquetas $63000 / \mathrm{mm}^{3}$. El PIM 2 score reveló $100 \%$ de posibilidad de fallecer. El score PELOD fue de 33. La radiografía de tórax fue compatible con una neumopatía bilateral. Posteriormente evolucionó con hipertermia de 39 ${ }^{\circ} \mathrm{C}$, hipotensión arterial persistente y aparición de ARDS $\left(\mathrm{PaO}_{2} / \mathrm{FiO}_{2} 70\right)$ asociada a acidosis respiratoria por lo que se inició ventilación de alta frecuencia oscilatoria e infusión de adrenalina ( $0,1 \mathrm{mcg} \mathrm{kg} \mathrm{min})$. A las cuatro horas de ingresada y previo consentimiento informado se decidió inducir HT usando el sistema Blanketrol ${ }^{\circledR}$ III Cincinatti Sub Zero (Cincinatti, $\mathrm{OH}, \mathrm{USA}$ ), dispositivo que requiere el uso de mantas térmicas por donde circula agua cuya $\mathrm{T}^{\circ}$ es regulada con ayuda de un servo mecanismo en función de la $\mathrm{T}^{\mathrm{o}}$ central del paciente, la que se mide con un sensor esofágico (figura 1). La caída de la $\mathrm{T}^{\mathrm{o}}$ por este sistema se produce por conducción. La inducción de la HT se realizó en alrededor de $12 \mathrm{~h}$, el período de mantención fue de 48 horas a $34^{\circ} \pm 0,5{ }^{\circ} \mathrm{C}$. Finalmente la fase de recalentamiento se realizó a una velocidad de 0,5 a $1{ }^{\circ} \mathrm{C}$ por hora en aproximadamente 12 horas hasta alcanzar la normotermia. En la figura 2 se muestra la evolución de la curva de $\mathrm{T}^{\mathrm{o}}$ esofágica de la paciente.

Una TAC cerebral no reveló signos de edema cerebral y un electroencefalograma se informó con lentitud difusa y sin actividad convulsiva.

A las 24 horas del ingreso la paciente evolucionó con estabilidad hemodinámica lo que permitió la suspensión de la adrenalina y la dismi- 


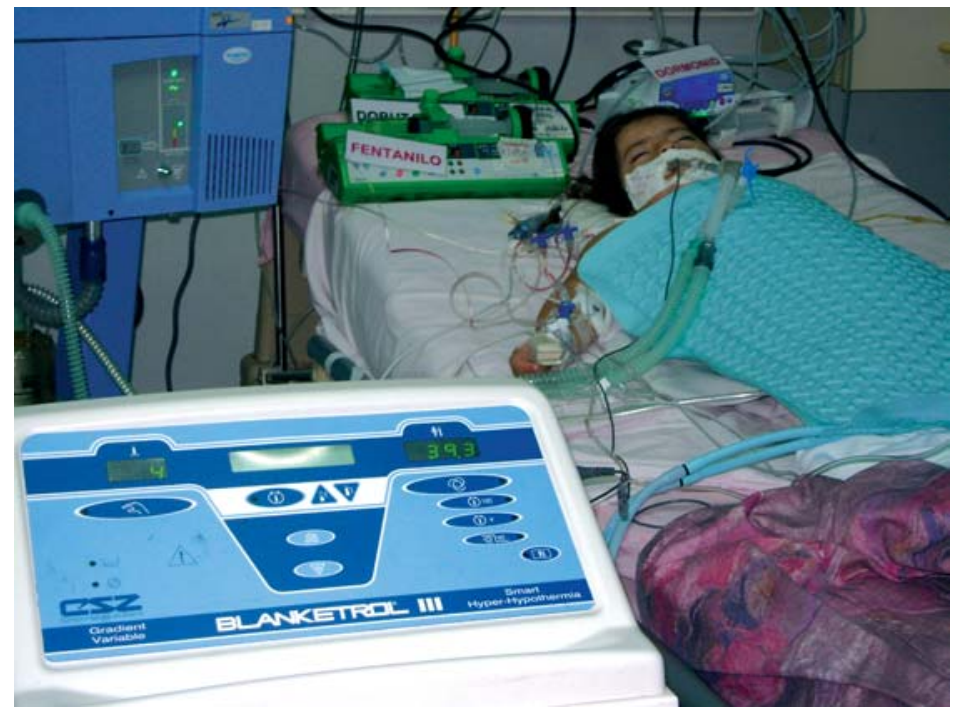

Figura 1. Sistema Blanketrol ${ }^{\circledR}$ III Cincinatti Sub Zero (Cincinatti, OH, USA) usado para la inducción de hipotermia en el caso presentado.

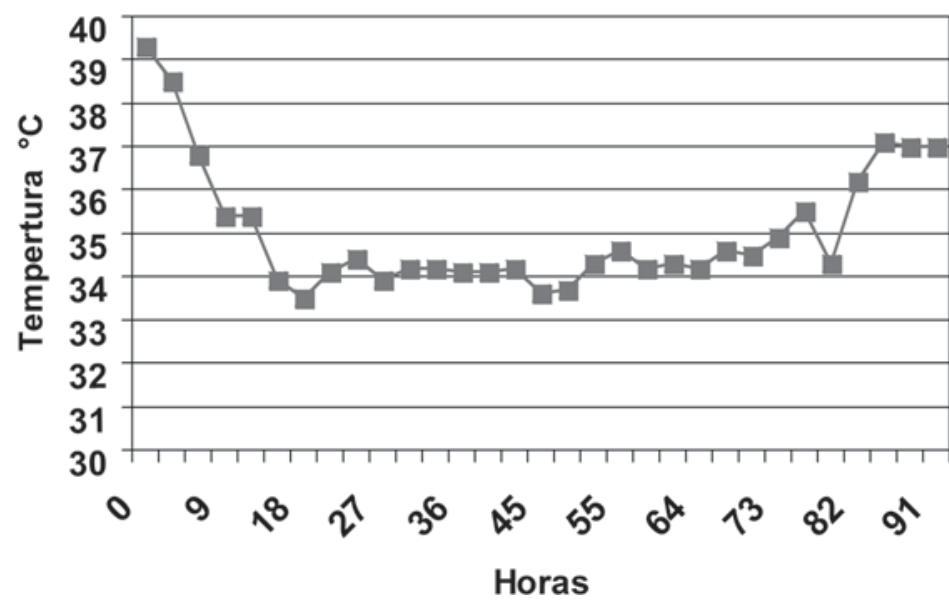

Figura 2. Evolución de la temperatura esofágica durante período de hipotermia. nución gradual de la dobutamina, normalización en el lactato sérico a $1,3 \mathrm{mmol} / \mathrm{L}$, con un EB $4,6, \mathrm{ScvO}_{2} 79 \%$ y glicemias en rango normal. Desde el punto de vista respiratorio se observó normalización en la ventilación y $\mathrm{PaO}_{2} / \mathrm{FiO}_{2}$ $>200$. La duración de la ventilación mecánica fue de 8 días y la estadía en UCIP fue de 10 días. Durante el procedimiento no se presentaron arritmias, infección nosocomial o hemorragia atribuibles a la terapia. Alta hospitalaria a los 20 días del ingreso motivada por infección intrahospitalaria de catéter central. La condición neurológica al alta hospitalaria estimada por el pediatric cerebral performance score (PCPC) fue de 2, similar al de la paciente previo su ingreso ${ }^{11}$ (Anexo 1).

\section{Discusión}

Este es el primer caso publicado en nuestro país de uso de HT en un paciente pediátrico con un PCR extrahospitalario. Se cree que la HT moderada suprime muchas de las reacciones químicas asociadas a la injuria por reperfusión. Sin embargo, se desconoce cuales son 
los mecanismos exactos mediante los cuales la hipotermia es beneficiosa para prevenir el daño cerebral en los humanos. La hipotermia en modelos experimentales animales produce entre otros: reducción del metabolismo cerebral (5-7\% por cada $\left.{ }^{\circ} \mathrm{C}\right)$, disminución de las demandas en zonas con flujo comprometido, reducción de la apoptosis que se desarrolla en las 48 horas postinjuria, actúa en la cascada excitotóxica que sigue a la isquemia y disminuye la liberación de mediadores inflamatorios ${ }^{12}$.

Como se menciona en la introducción, existe evidencia sólida de los efectos benéficos de la hipotermia moderada en pacientes adultos y recién nacidos con EHI. El International Liaison Committee on Resuscitation (ILCOR) recomienda que "pacientes adultos inconscientes con circulación espontánea después de un PCR extrahospitalario deberían ser enfriados entre $32{ }^{\circ} \mathrm{C}$ y $34^{\circ} \mathrm{C}$ por 12 a 24 horas cuando el ritmo inicial sea la fibrilación ventricular" y que el "enfriamiento podría tener beneficios para otros ritmos o en el PCR intrahospitalario"13. También se han reportado experiencias preliminares en adultos con infarto cerebral, falla hepática con encefalopatía e hipertensión endocraneana y en casos de infarto al miocardio ${ }^{14-15}$.

A diferencia de la población adulta y neonatal, la experiencia con HT en niños con PCR es limitada y no se han conducido ensayos randomizados en este escenario en pediatría, por lo que las guías más recientes de la American Heart Association clasifican a la hipotermia inducida post paro sólo como recomendación IIB en niños ${ }^{16}$.

Una encuesta realizada a intensivistas pediátricos en USA revelo que sólo un 5\% usa de manera habitual y un 38\% ocasionalmente esta terapia en niños con PCR, lo que podría explicarse por varias razones, en primer lugar: la mayoría de la evidencia publicada en adultos es en pacientes con paro cardíaco en fibrilación ventricular, experiencia que no puede ser extrapolada a los niños. Lo mismo ocurre con la asfixia perinatal situación donde los recién nacidos con asfixia no se presentan en paro cardíaco sino más bien hipodébito asociado a hipoxia de diferente intensidad, de tal modo que es una población no comparable al PCR pediátrico. Por otro lado, no existen hasta la fecha guías que recomienden el uso de la HT en el PCR pediátrico y por último no hay un consenso sobre el método más seguro y efectivo de inducir hipotermia, que pacientes deberían ser enfriados, la manera de monitorizar la $\mathrm{T}^{\mathrm{o}}$, la profundidad y duración del enfriamiento ${ }^{17}$. Algunas de estas interrogantes podrían ser resueltas con los resultados del estudio multicéntrico randomizado auspiciado por el National Heart Lung and Blood Institute de Estados Unidos llamado "Therapeutic Hypotermia After Pediatric Cardíac Arrest" donde se evaluará el efecto de la HT a $32-34^{\circ} \mathrm{C}$ durante 48 horas en el PCR pediátrico de origen extra e intrahospitalario $^{18}$.

En nuestro caso, el inicio de la HT se realizó en un plazo aceptable ya que la mayoría sugiere que su aplicación debe ser antes de las 6 horas de transcurrido el evento hipoxico isquémico. El tiempo de inducción de la HT fue más lento que lo descrito en la literatura ya que la mayoría de los expertos sugieren que debe realizarse lo más rápidamente posible y desde la sala de urgencia, lo anterior debido probablemente a la inexperiencia de nuestro grupo con esta terapia.

Respecto a los métodos disponibles para la inducción de hipotermia un estudio prospectivo comparativo demostró que el uso de mantas térmicas y catéteres intravasculares son más eficientes que los métodos convencionales (bolsas de hielo e infusión de soluciones salinas frías) o los sistemas de enfriamiento de aire para lograr la $\mathrm{T}^{\mathrm{o}}$ deseada. En este estudio, el uso de catéteres intravasculares permitió la mantencion de la $\mathrm{T}^{\mathrm{o}}$ en un rango más estrecho respecto a la $\mathrm{T}^{\mathrm{o}}$ deseada. En términos de costos, el uso de catéteres acarrea costos más elevados en relación a los otros métodos ${ }^{19}$.

Se han reportado efectos adversos derivados de esta técnica, lo más frecuente es que ocurran en la fase de inducción de la hipotermia como la hipovolemia que lleva a la necesidad de aporte de volumen y la aparición de temblores que pueden ser prevenidos o tratados con adecuada analgesia, sedacion, sulfato de magnesio o un agente paralizante de acción corta. También se han descrito arritmias cardíacas, predisposición a infecciones, hiperglicemia, trastornos hidroelectroliticos y de la coagulación. 
La hipotermia produce una disminución en el "clearence" de algunas drogas, en la mayoría de los casos aumentando los niveles y el efecto de drogas como vasopresores, opiáceos y sedantes lo que podía explicar el rápido retiro de la dobutamina y adrenalina en nuestra paciente así como la prolongación de la ventilación por el efecto de acumulación de la sedo-analgesia ${ }^{20}$.

Si bien es aceptado que la hipertermia puede exacerbar el daño neurológico después de un evento hipóxico isquémico y en este contexto el intensivista debe realizar intervenciones para restaurar la normotermia lo más pronto posible ${ }^{21-22}$, debe destacarse que la HT usada en el contexto de PCR en niños permanece una terapia experimental y que en el caso presentado fue usada como medida extraordinaria dada las altas posibilidades de fallecer e importante disfunción multiorgánica expresada en el score PIM 2 y PELOD al ingreso de la paciente a la UCIP.

\section{Conclusión}

Si bien esta establecido el rol neuroprotector de la hipotermia terapéutica en recién nacidos con encefalopatía hipóxico isquémica y en adultos en PCR con fibrilación ventricular y asistolia, en pacientes pediátricos no existe evidencia de su eficacia por lo que los ensayos randomizados actualmente en curso podrán establecer el lugar de esta terapia en medicina intensiva pediátrica.

Anexo 1. Descripción de la escala de discapacidad PCPC (Pediatric Cerebral Performance Category)

\begin{tabular}{|c|c|c|}
\hline Puntuación & Categoría & Escala \\
\hline 1 & Normal & $\begin{array}{l}\text { Normal para la edad } \\
\text { Acude a escuela en curso que le corresponde }\end{array}$ \\
\hline 2 & Leve & $\begin{array}{l}\text { Consciente alerta y capaz de interactuar para la edad } \\
\text { Acude a escuela nivel inferior para la edad } \\
\text { Trastorno neurológico menor }\end{array}$ \\
\hline 3 & Moderada & $\begin{array}{l}\text { Consciente función por debajo de su edad enfermedad neurológica no } \\
\text { controlada que limita sus actividades } \\
\text { Realiza actividades de vida diaria de modo independiente } \\
\text { Déficit de aprendizaje yo necesidad de escuela especial }\end{array}$ \\
\hline 4 & Grave & $\begin{array}{l}\text { Consciente dependiente de otros para la vida diaria } \\
\text { Incapaz de acudir a la escuela }\end{array}$ \\
\hline 5 & $\begin{array}{l}\text { Coma o estado } \\
\text { vegetativo persistente }\end{array}$ & $\begin{array}{l}\text { Cualquier grado de coma } \\
\text { Ausencia de respuesta cerebral y signos de función de la corteza } \\
\text { Posibilidad de alguna respuesta refleja ciclo sueño vigilia apertura espontánea } \\
\text { de ojos }\end{array}$ \\
\hline 6 & Muerte cerebral & Signos de muerte cerebral \\
\hline
\end{tabular}

\section{Referencias}

1.- Donoghue AJ, Nadkarni V, Berg RA, et al: Out-ofhospital pediatric cardiac arrest: an epidemiologic review and assessment of current knowledge. Ann Emerg Med 2005; 46: 512-22.

2.- Young KD, Gausche-Hill M, McClung CD, et al: A prospective, population-based study of the epidemiology and outcome of out-of hospital pediatric cardio- pulmonary arrest. Pediatrics 2004; 114: 157-64.

3.- Nadkarni VM, Larkin GL, Peberdy MA, et al; for the National Registry of Cardiopulmonary Resuscitation Investigators: First documented rhythm and clinical outcome from in-hospital cardiac arrest among children and adults. JAMA 2006; 295: 50-7.

4.- Pinochet C, Donoso A, León J, Urioste J, Arriagada $D$ : Paro cardiorrespiratorio extrahospitalario. Hospital Padre Hurtado. Análisis clínico-epidemiológico. Pe- 
ríodo 2000-2008. Libro de resúmenes VI Congreso Chileno de Cuidados Intensivos en Pediatría Chillán 2008.

5.- Schindler $M B$, Bohn DB, Cox PN, et al: Outcome of out-of-hospital cardiac or respiratory arrest in children. N Engl J Med 1996; 335: 1473-9.

6.- Bernard SA, Gray TW, Buist MD, et al: Treatment of comatose survivors of out-of hospital cardiac arrest with induced hypothermia. N Engl J Med 2002; 346: 557-63.

7.- The Hypothermia After Cardiac Arrest Study Group: Mild therapeutic hypothermia to improve the neurologic outcome after cardiac arrest. N Engl J Med 2002; 346: 549-56.

8.- Shankaran S, Laptook AR, Ehrenkranz RA, et al, for the National Institute of Child Health and Human Development Neonatal Research Network: Whole-body hypothermia for neonates with hypoxic-ischemic encephalopathy. N Engl J Med 2005; 353: 1574-84.

9.- Gluckman PD, Wyatt JS, Azzopardi D, et al: Selective head cooling with mild systemic hypothermia after neonatal encephalopathy: multicentre randomised trial. Lancet 2005; 365: 663-70.

10.- Eicher DJ, Wagner CL, Katikaneni LP, et al: Moderate hypothermia in neonatal encephalopathy: efficacy outcomes. Pediatr Neurol 2005; 32: 11-7.

11.- Fiser D: Assessing the outcome of pediatric intensive care. J Pediatr 1992; 121: 68-74.

12.- Polderman $K H$ : Induced hypothermia and fever control for prevention and treatment of neurological injuries. Lancet 2008; 371: 1955-69.

13.- Nolan JP, Morley PT, Vanden Hoek TL, et al: Therapeutic hypothermia after cardiac arrest: an advisory statement by the advanced life support task force of the International Liaison Committee on Resuscitation. ILCOR. Circulation 2003; 108 (1): 118-21.
14.- Jalan R, Olde Damink SW, Deutz NE, Hayes PC, Lee $A$ : Moderate hypothermia in patients with acute liver failure and uncontrolled intracranial hypertension. Gastroenterology 2004; 127: 1338-46.

15.- Dixon SR, Whitbourn RJ, Dae MW, et al: Induction of mild systemic hypothermia with endovascular cooling during primary percutaneous coronary intervention for acute myocardial infarction. J Am Coll Cardiol 2002; 40: 1928-34.

16.- American Heart Association: Guidelines for emergency cardiovascular care and cardiopulmonary resuscitation. Part 12: Pediatric Advance Life Support. Circulation 2005; 112 (24 Suppl): IV-167-87.

17.- Haque IU, Latour MC, Zaritsky AL: Pediatric critical care community survey of knowledge and attitudes toward therapeutic hypothermia in comatose children after cardiac arrest. Pediatr Crit Care Med 2006; 7: 714.

18.- Therapeutic Hypotermia After Pediatric Cardiac Arrest: disponible en: http://clinicaltrials.gov/ct2/show/ NCT00880087term=hypothermia. Ingreso el 9 de julio 2009.

19.- Hoedemaekers CW, Ezzathti M,Gerritsen A, Van der Hoeven JG: Comparison of cooling methods to induce and maintain normo and hypothermia in intensive care unit patients:a prospective intervention study. Crit Care 2007; 11: R91.

20.- Polderman K H, Herold I: Therapeutic hypothermia and controlled normothermia in the intensive care unit: Practical considerations, side effects, and cooling methods. Crit Care Med 2009; 37: 1101-20.

21.- Kleinman ME, Srinivasan V: Postresuscitation Care. Pediatr Clin N Am 2008; 55: 943-67.

22.- Hutchison JS, Doherty DR, Orlowski JP, Kissoon N: Hypothermia Therapy for Cardiac Arrest in Pediatric Patients. Pediatr Clin N Am 2008; 55: 529-44. 